less sensitive than DAS28 for detecting low disease activity. PDAS1 and 2 also correlated well with a range of disease activity measures, but weakly with generic health measures. The two measures were comparable with DAS28 for sensitivity to changes in activity, as assessed in a cohort of 56 patients after 6 months of treatment.

The authors suggest that PDAS assessment improves patients' involvement with their disease and could be used in clinical practice and trials.

Original article Choy EH et al. (2008) Development and validation of a patient-based disease activity score in rheumatoid arthritis that can be used in clinical trials and routine practice. Arthritis Rheum 59: 192-199

\section{Mouse studies shed light on pathogenesis of Lyme arthritis}

Lyme arthritis develops (weeks or months after the original tick bite) in a substantial number of untreated individuals infected with Borrelia burgdorferi, following spirochete colonization of joints. $\mathrm{C} 3 \mathrm{H} \times \mathrm{HeN}$ mice are used to model Lyme arthritis: in these mice, arthritis develops $\sim 10$ days after infection with $B$. burgdorferi, reaches peak disease activity after 2-3 weeks, then diminishes and resolves by 8 weeks after infection.

$\mathrm{Pal}$ and colleagues used microarrays to compare the expression levels of $B$. burgdorferi genes in spirochetes from various sources. The authors discovered that basic membrane proteins $A$ and $B$ (bmpA and $b m p B$ ) were preferentially upregulated in spirochetes isolated from joints, compared with spirochetes from other mouse tissues and from ticks. Expression of bmpA and bmpB was greatest 12-15 days after infection. Passive transfer of monospecific antibodies to $\mathrm{bmpA}$ and $\mathrm{bmpB}$ did not eradicate infection but dramatically reduced spirochete colonization and swelling of mouse joints, and somewhat reduced skin colonization. Spirochetes engineered to lack both bmpA and bmpB genes were as infectious as wild-type organisms, but did not colonize mouse joints or cause arthritis. Furthermore, bmpA and bmpB were highly expressed in spirochetes from synovial fluid of patients with acute Lyme arthritis.

$\mathrm{Pal}$ and colleagues infer that bmpA and bmpB have essential roles in colonization and persistence of $B$. burgdorferi within joints. Understanding the adaptive strategies used by this pathogen might lead to new ways to modulate Lyme arthritis.

Original article Pal U et al. (2008) Borrelia burgdorferi basic membrane proteins $A$ and $B$ participate in the genesis of Lyme arthritis. J Exp Med 205: 133-141

\section{Duloxetine treatment is effective in women with fibromyalgia}

Dysfunction of serotonin and norepinephrine signaling is a possible cause of fibromyalgia. Arnold et al. report the results of a pooled analysis of two trials of duloxetine, a serotonin and norepinephrine reuptake inhibitor, in women with fibromyalgia. Compared with placebo, duloxetine significantly reduced the pain and functional impairment associated with fibromyalgia.

The combined study population comprised 538 women, 326 of whom received duloxetine (60 mg either daily or twice daily) for 12 weeks and 212 received placebo. Women who received duloxetine had significantly greater improvement on the Brief Pain Inventory and the Fibromyalgia Impact Questionnaire than did those who received placebo $(P<0.001)$. Duloxetine also improved their quality of life, measured by the Medical Outcomes Study Short Form 36 questionnaire. Duloxetine was equally effective in women with and without major depression. However, 90 women stopped treatment because of adverse effects: 68 duloxetine-treated patients $(20.9 \%)$ and 22 placebo-treated patients (10.4\%). Nausea, insomnia and headache were the most commonly reported adverse effects. There were two serious adverse events in duloxetinetreated patients: one case of appendicitis and one of increased liver enzyme levels.

The authors conclude that 12 weeks of duloxetine treatment is safe and effective in women with fibromyalgia. Longer-term studies are required to determine whether this effect can be maintained. One trial included 23 male patients, in whom duloxetine was ineffective; further studies are needed to investigate the efficacy of this treatment in men.

Original article Arnold LM et al. (2007) Duloxetine for the treatment of fibromyalgia in women: pooled results from two randomized, placebo-controlled clinical trials. J Womens Health (Larchmt) 16: 1145-1156 